Journal of Mathematics and Informatics

Vol. 17, 2019, 67-72

ISSN: 2349-0632 (P), 2349-0640 (online)

Published 27 July 2019

www.researchmathsci.org

DOI: http://dx.doi.org/10.22457/jmi.150av17a6

Journal of

Mathematics and

Informatics

\title{
Difference Analysis of the Influence of Game Categories on Audience Engagement
}

\author{
Chong-Li Wang ${ }^{1}$, Hong Chen ${ }^{2}$, Hua-Qiu Liang ${ }^{3}$ and Jing Liu ${ }^{4}$ \\ ${ }^{1}$ School of Economics and Management \\ Chongqing University of Posts and Telecommunications \\ Chongqing - 400065, Chongqing, China. E-mail: 1763256864@qq.com \\ ${ }^{2}$ School of Economics and Management \\ Chongqing University of Posts and Telecommunications \\ Chongqing - 400065, Chongqing, China. E-mail: 1159878308@qq.com \\ ${ }^{3}$ School of Economics and Management \\ Chongqing University of Posts and Telecommunications \\ Chongqing - 400065, Chongqing, China. E-mail: $964476230 @ q q . c o m$ \\ ${ }^{4}$ Information and Library Center \\ Chongqing Medical and Pharmaceutical College \\ Chongqing - 401331, Chongqing, China. E-mail: 717139038@qq.com \\ ${ }^{4}$ Corresponding author
}

Received 12 June 2019; accepted 26 July 2019

Abstract. With the popularity of online video game, online video has become one of the most popular online video. By crawler technology, this paper obtained 2,272 data related to audience participation (View, Follow, Barrage,Thumb up, Reward and Collect). Then, based on Kendall correlation and one-way ANOVA, this paper explores the influence of three types of game videos (PC game, e-sports, online game) on audience engagement. It is found that the influence of game categories on audience participation is different, and PC games video has the highest view counts, number of followers, number of barrage screen, number of thumb up, reward amount and collection amount among the three types of game videos.

Keywords: Game video; Audience engagement; Web crawlers; One-way ANOVA

AMS Mathematics Subject Classification (2010): 14C21

\section{Introduction}

With the advent of web2.0, Chinese network video industry has developed rapidly, and many video websites have appeared successively, including Youku, Tencent, iQIYI, Bilibili and so on. The number of Chinese video users those who watched or downloaded video online in the past six months had reached 612 million by December 2018, 33.09 million more than the end of 2017 , accounting for $73.9 \%$ of the total internet users [1]. Among them, game online video (hereinafter referred to as game video) is one of the most popular online video [2]. On the video website, audiences can view at any time 
Chong-li Wang, Hong Chen, Hua-qiu Liang and Jing Liu

without any limit on playing times and playing time, and they can watch videos while downloading [3]. The viewers also can collect game video, thumb up game video, send the barrage, follow up Lord and reward up Lord.

This paper obtained 2272 pieces of data related to videos of three main games on bilibili website by crawler technology. Firstly, Kendall correlation coefficient was used to analyze the correlation between game categories and audience participation index. Then, the statistical method of one-way analysis of variance was used to explore whether game types affect the engagement indicators of audience behavior --view, collect, thumb up, barrage, follow and reward. Finally, the conclusions and limitations of this paper were summarized.

\section{Theoretical basis and hypothesis}

\subsection{Analysis methods}

Kendall correlation coefficient is used to explore the correlation between variables, and no assumptions need to be made about the relationship between variables in the initial stage. Among them, the correlation coefficient (hereinafter referred to as " $\mathrm{t}$ ") scope is:

$[-1,1], \mathrm{t}>0$ means two variables are correlation, $\mathrm{t}<0$ means two variables against correlation; $\mathrm{t}=0$ means two variables are independent of each other. The higher the value of $|\mathrm{t}|$ is, the higher the correlation between the two variables is.

Analysis of Variance (ANOVA) is used to test the significance of differences in the mean values of two or more samples and then to judge whether the influence of each factor on the test index is significant. According to the number of factors affecting the test index conditions, it can be divided into one-way ANOVA, two-factor ANOVA and multi-factor ANOVA. This paper only considers whether there are differences in the influence of game types on viewer engagement indexes such as view count, followers number, barrage amount on game network video. Therefore, this study adopts one-way ANOVA to explore the influence of game types on viewer engagement.

\subsection{Research hypothesis}

Hypothesis 1: there are differences in the influence of PC games, e-sports games and online games on view count.

Hypothesis 2: there are differences in the influence of PC games, e-sports games and online games on followers amount.

Hypothesis 3: there are differences in the influence of PC games, e-sports games and online games on barrage amount.

Hypothesis 4: there are differences in the influence of PC games, e-sports games and online games on thumb up amount.

Hypothesis 5: there are differences in the influence of PC games, e-sports games and online games on reward amount.

Hypothesis 6: there are differences in the influence of PC games, e-sports games and online games on collection amount. 
Difference Analysis of the Influence of Game Categories on Audience Engagement

\section{Data analysis}

\subsection{Research design}

Bilibili is a famous video barrage website in China, which has a special game zone. The video website is convenient for directly collecting the viewer engagement indicator data of the classified game video, such as the collection of view count, barrage amount, thumb up amount and so on. In this study, network crawler technology was adopted to crawl 3000 video data of "bilibilii" website in nearly one month of June 2019. Among them, 179 missing data were deleted and 2821 valid data were collected. In this paper, the above data are summarized based on classification tags of game video. It is found that three types of game video labeled as "PC games, e-sports games and online games" has a large number, including 2272 pieces of data. Therefore, this research focuses on whether these three types of game video has different influences on audience engagement indicators.

Data collected in this study include the following indicators: view count, barrage amount, thumb UP amount, reward amount, collection amount, and the number of followers of the video UP master. The sample description statistics are shown in Table 1.

Then, three kinds of game videos are selected according to the game category label: PC game video, e-sports video, online game video. The number of PC games video is the largest among them, accounting for 55\% of the total number of three types of game videos. The amount of e-sport game video accounts for $26.2 \%$. The amount of online game video accounts for $26.2 \%$.

Table 1: Descriptive statistics

\begin{tabular}{llclll}
\hline & N & Minimum & Maximum & Mean & Std. Deviation \\
\hline View & 2272 & 4 & 4366000 & 254236.847 & 500263.574 \\
Follow & 2272 & 1 & 5475000 & 490615.956 & 935256.554 \\
Barrage & 2272 & 0 & 1029000 & 6217.647 & 32735.480 \\
Thumb up & 2272 & 1 & 393000 & 7612.628 & 21072.651 \\
Reward & 2272 & 1 & 1428000 & 8150.253 & 37960.109 \\
Collect & 2272 & 1 & 180000 & 3934.598 & 10985.664 \\
\hline
\end{tabular}

\subsection{Data analysis}

Firstly, this paper analyzes the correlation between game categories and view count, followers amount, barrage, thumb up amount, reward amount and collection amount by Kendall's tau_b. The correlation between game classification and its engagement indicators is significant at the level of 0.05, indicating that these three types of games are correlated with viewer engagement. Correlation analysis results are shown in Table 2.

Secondly, based on the correlation between the two, we adopted the one-way variance method to explore whether there is significant difference in the influence of game categories on viewer engagement. The significance probability was $\mathrm{p}<0.05$, indicating that the mean value of each group was significantly different at the 0.05 level.Analysis of Variance is shown in Table 2. 
Chong-li Wang, Hong Chen, Hua-qiu Liang and Jing Liu

Table 2: Correlation, ANOVA \& Test of Homogeneity

\begin{tabular}{lllllll}
\hline & Game Category & & \multicolumn{2}{c}{ ANOVA } & \multicolumn{3}{c}{$\begin{array}{c}\text { Test of Homogeneity } \\
\text { of Variances }\end{array}$} \\
\hline & $\begin{array}{l}\text { Correlation } \\
\text { Coefficient }\end{array}$ & Sig. & F & Sig. & $\begin{array}{l}\text { Levene } \\
\text { Statistic }\end{array}$ & Sig. \\
\hline View & $-.078^{* *}$ & .000 & 8.148 & .000 & 14.547 & .000 \\
Follow & $-.126^{* *}$ & .000 & 25.275 & .000 & 64.263 & .000 \\
Barrage & $-.145^{* *}$ & .000 & 7.824 & .000 & 15.608 & .000 \\
Thumb up & $-.158^{* *}$ & .000 & 5.490 & .004 & 5.008 & .007 \\
Reward & $-.187^{* *}$ & .000 & 6.256 & .002 & 10.525 & .000 \\
Collect & $-.204^{* *}$ & .000 & 16.638 & .000 & 29.192 & .000 \\
\hline
\end{tabular}

Then we conducted the homogeneity test of variance. The significance probability is $\mathrm{p}<0.05$, indicating that the variance of each group has significant difference at the level of 0.05 , that is, the variance is not homogeneous. Test of homogeneity of variances is shown in Table 2.

When variance is not homogeneous, we test multiple comparisons of "unassumed homogeneity of variance" with Tamhane's T2. The result of Multiple Comparisons is shown in Table 3.

Table 3: Multiple Comparisons

\begin{tabular}{|c|c|c|c|c|}
\hline $\begin{array}{l}\text { Multiple } \\
\text { Comparisons }\end{array}$ & $\begin{array}{l}\text { (I)Game } \\
\text { Category }\end{array}$ & $\begin{array}{l}\text { (J)Game } \\
\text { Category }\end{array}$ & $\begin{array}{l}\text { Mean } \\
\text { Difference (I-J) }\end{array}$ & Sig. \\
\hline View & online game & $\begin{array}{l}\text { single game } \\
\text { e-sport game }\end{array}$ & $\begin{array}{l}-110582.233^{*} \\
-63276.697^{*}\end{array}$ & $\begin{array}{l}.000 \\
.044\end{array}$ \\
\hline Follow & online game & $\begin{array}{l}\text { single game } \\
\text { e-sport game }\end{array}$ & $\begin{array}{l}-367027.347^{*} \\
-309764.851^{*}\end{array}$ & $\begin{array}{l}.000 \\
.000\end{array}$ \\
\hline Barrage & single game & $\begin{array}{l}\text { online game } \\
\text { e-sport game }\end{array}$ & $\begin{array}{l}6490.905^{*} \\
4376.755\end{array}$ & $\begin{array}{l}.000 \\
.077\end{array}$ \\
\hline Thumb up & PC game & $\begin{array}{l}\text { e-sport game } \\
\text { online game }\end{array}$ & $\begin{array}{l}2674.344 * \\
3248.171^{*}\end{array}$ & $\begin{array}{l}.004 \\
.037\end{array}$ \\
\hline Reward & single game & $\begin{array}{l}\text { e-sport game } \\
\text { online game }\end{array}$ & $\begin{array}{l}6396.420^{*} \\
4110.034\end{array}$ & $\begin{array}{l}.000 \\
.120\end{array}$ \\
\hline Collect & single game & $\begin{array}{l}\text { e-sport game } \\
\text { online game }\end{array}$ & $\begin{array}{l}3079.266^{*} \\
1626.541\end{array}$ & $\begin{array}{l}.000 \\
.068\end{array}$ \\
\hline
\end{tabular}

View: online game video and PC game video, online game video and sports game video have differences in view counts at the level of 0.05 . The average difference between online game video and PC game video is -110582.233 , and that between online game video and e-sports video is -63276.697 . As can be seen from the average amount of view count, PC game video have the highest amount of view count, followed by e-sports video, and then online game video.

Follow: online game video and PC game video, online game video and sports game 
Difference Analysis of the Influence of Game Categories on Audience Engagement

video have differences in followers amount at the level of 0.05 . The average difference between online game video and PC game video is -367027.347 , and that between online game video and e-sports video is -309764.851. As can be seen from the average amount of view counts, online game video have the highest followers amounts, followed by esports video, and then PC game video.

Thumb up: PC game video and e-sports game video, PC game video and online game video have differences in followers amount at the level of 0.05 . The average difference between PC games video and e-sports game video is 2674.344, and that between PC game video and online game video is 3248.171. As can be seen from the average amount of view counts, online game video have the highest Thumb up amount, followed by e-sports video, and PC then game video.

Others: at the level of 0.05 , only PC game video and online game video have significant differences in the number of barrage. For the average number of barrage, only e-sports video and PC game video have significant differences in the reward amounts. Finally, only PC game video and e-sports video have differences in the average number of collection.

\section{Conclusion and discussion}

\subsection{Conclusion}

For PC games, e-sports games and online games, there are differences in viewer engagement indexes, such as view count, followers amount, barrage amount, thumb up amount, reward amount and collection amount, etc. And these three games types affect the participation of online video viewers. In addition, PC game video has the highest view count, number of followers, number of barrage screen, number of thumb up, reward amount and collection amounts among the three types of games video. E-sports video is the lowest in the number of rewards and collections. Online games have the lowest audience participation indexes in view, followers, barrage, and thumb up.

\subsection{Suggest}

For video website and up host, if they want to have more audience participation, they could choose to upload related PC game video. PC game video now accounts for more than half of all videos of the three categories, and is far ahead of the other two in user engagement. In addition, e-sports video could also be selected, because the popularity of e-sports video continues to rise [9].

\subsection{Research limitations}

The data collection method has some defects. This study only crawls the static data of "bilibilii" web page, which cannot fully reflect audience participation. Because the information mined from the content server is not exactly the same as the information mined from the network nodes. For example, there are inconsistencies in video length and viewing time, encoding rate and download rate. Static information on the server cannot fully reflect the dynamic behavior characteristics of users [10]. Later research can compare and analyze the data mined from the server and the network nodes.

The research content is too single. This paper only explores the differences in the impacts of the three types of games on viewer engagement. After that, we can explore the influence of various types of games on audience participation. Future research could also 
Chong-li Wang, Hong Chen, Hua-qiu Liang and Jing Liu

explore whether the length of video has a differentiated impact on audience engagement indicators. Moreover, a questionnaire survey can be used to investigate the audience on video website. So we could analyze the differences between audience engagement and their preference for video types of games from the perspective of users.

Acknowledgment. This work is supported by the Undergraduate Scientific Research Training Program of Chongqing University of Posts and Telecommunications(K2019107).

\section{REFERENCES}

1. The 43rd statistical report on Internet development in China, China Internet network information center, 2019.

2. F.Bentley, M.Silverman and M.Bica, Exploring online video watching behaviors, Proceedings of the 2019 ACM International Conference on Interactive Experiences for TV and Online Video, (2019) 108-117.

3. Y.Wang, Research on video vod system user life cycle and video active period, Beijing Jiaotong University, 2018.

4. 2018 research report on online audio-visual development in China, Office of the central network security and information technology commission of the CPC, 2019.

5. X.F.Tang, Changes in bullet screens, scenes and social roles, Fujian BBS: humanities and social sciences edition, 12 (2015) 139-145.

6. L.S.Zhang and X.Y.Ge, The change of network video users' viewing habits under the popular background of barrage, Contemporary Communication, 49 (3) (2018) 93-95.

7. L.Y.Liu and J.F.Wang, Design research of video viewing experience elements of bullet screen, Industrial design research, (2018) 23.

8. Y.J.Hu and G.M.Yu, Research on the behavior pattern of video users in Chinese network, Journal of Zhejiang University of Communication, 2 (2018) 9-17.

9. B.C.B.Churchill and W.Xu, The modem nation: A first study on Twitch. tv social structure and player/game relationships, 2016 IEEE International Conferences on Big Data and Cloud Computing (BDCloud), Social Computing and Networking (SocialCom), Sustainable Computing and Communications (SustainCom)(BDCloudSocialCom-SustainCom). IEEE, (2016) 223-228.

10. H.B.Lu and Y.G.Qian, Information mining based on YouTube video sharing system, Journal of Zhejiang University of Science and Technology, 250 (3) (2018) 230-234. 